\title{
EDITORIAL
}

\section{Complex systems in composition and improvisation}

This issue presents a contemporary perspective on the role complex adaptive systems can play in the understanding, generation and analysis of sound structures for composition and improvisation.

A complex system is a structure whose behaviour cannot be extrapolated from the behaviours of its individual components. A large number of such systems can be found in fields as diverse as particle physics, ecology, economics, neurology, sociology and computer science. Their behaviour cannot be controlled or designed in a hierarchical way and they evolve unusual characteristics as they interact with and adapt to the environment in which they operate.

Yet, despite this complex reciprocity between each structure and its environment, some largely context-independent fundamental concepts have been found which have enabled a shift in approach to the design of systems, including musical ones. A common vocabulary and set of characteristics has evolved along with a theoretical understanding which acknowledges the need to balance descriptions on a system level with descriptions on a component level. Furthermore, the development of this common vocabulary has enabled researchers from diverse backgrounds to engage in multidisciplinary attempts to explore and control the simultaneous behaviour of such systems on all of their levels and sub-levels.

Complex systems share a number of characteristics, the most common of which are:

- Self-organisation into critical states ranging from stable to chaotic.

- Emergence of behaviours such as phase transitions and bifurcations resulting in spontaneous pattern formation and self-similarity under power laws (fractals and chaotic attracters, for example).

- Robustness - they are adaptable to external influences rather than being resistant or overwhelmed, often exchanging energy with their environment.

The most successful applications of complex systems so far have been in pattern recognition, so it is perhaps not surprising that there are practical applications for organising sound. Their use has been well embedded in contemporary computer animation practice for quite some time now, for the generation of such visual effects as fire, ocean waves, as well as schooling, flocking, herding and swarming. Many insects exhibit such self-organisation, and Tim Blackwell and Michael Young's paper reports on their ongoing work in applying the principles of stigmergy, the process by which social insects communicate indirectly by modifying their local environment, to a human-machine improvising music system with similar characteristics.

Bret Battey explores the use of a variable-coupled iterated-map network of nodes to produce richly gestural musical structures. For this work, Battey has used Derrick Lehmer's Linear Congruence Formula, which is widely employed as a pseudo-random sequence generator. The nodes are interlinked with time-delays to produce self-modulating musical patterns. He discusses some of the difficulties that arise when working with nonlinear systems, including the still quite limited number of tools available and the continued need for a trial-and-error approach to 'tuning' such generative systems.

As I mentioned earlier, our understanding of patterns and patterning has benefited greatly from research into complex systems, and this is resulting in a set of principles for the construction of generative models from information which is embedded in natural sound and image forms. Yon Visell began his work on spontaneous organisation and pattern models for interactive computer music composition from a self-organising hidden Markov model to represent the spontaneous organisation of sound events. Many of the readers of this journal will be familiar with Iannis Xenakis' groundbreaking use of Markovian stochastics in his music, and will recognise Visell's work as a fecund and contemporary extension to it.

Some may think it strange that a topic that appears so theoretical has so many direct practical applications in interactive music-making. Ian Whalley describes the infrastructure and aesthetic approach he used in his Public Space Interactive Web-Based Composition System which uses a multi-agent technology, together with short performed samples of traditional New Zealand Maori musical instruments as the basis of a system for sustaining musical conversations which can be initiated and extended through electroacoustic manipulation of the source material. 
Attempts to forecast the weather more accurately have played a central role in complexity studies. Trying to better perceive the interrelations between the components of such a multi-variate atmospheric system is the subject of Andrea Polli's paper in which she reports on an interdisciplinary project to produce spatial sonifications for an installation of weather data for both musical and scientific purposes.

Terry Bossomaier and Allan Snyder's paper turns the problem of music pattern perception on its head by reporting on their research into ways of temporarily switching off part of the brain in order to access 'Absolute Pitch'. This concerns an ability which, it seems, unlike birds and other animals, most of us lost during early maturation. Using agency-based modelling techniques, they have developed a computerised neural network for exploring the rationale for such inhibitory processes in the brain. This work sheds light on a number of issues related to music perception and composition, including ways of listening to musique concrète, as well as suggesting techniques for modelling compositional tools in congested situations such as real-time interactive sonic environments.

Andrew Brown's study in genetically evolving melodies takes us back into the realm of high-level pattern recognition and selection. Based on the earlier model of Richard Dawkin's evolving stick insects, Brown uses a genetic algorithm technique to evolve new melodies from older ones, then selects those that are to survive into the next generation by assigning each melody an aesthetic value.

Two articles not on the theme of this issue complete the papers section. Helen Metzelaar writes on the participation of women improvisers in the Netherlands, and suggests that a different network model exits for men and women participants, whilst Phil Thompson summarises a number of the technical issues involved in microsound composition and analysis, and examines some aesthetic implications in a social context.

This edition was prepared by Mitchell Whitelaw and myself, ably assisted by Organised Sound's Editor, Leigh Landy. My thanks go to them for making the process both efficient and enjoyable.

David Worrall Sonic Communications Research Group University of Canberra David.Worrall@canberra.edu.au Mitchell.Whitelaw@canberra.edu.au 